\title{
Entre la "salud" y la "tradición": las representaciones sociales de la dieta mediterránea
}

I ${ }^{1}$ Maria Clara de Moraes Prata Gaspar, ${ }^{2}$ Úrsula Verthein I

Resumen: El presente estudio analiza, a través de una aproximación cualitativa, las representaciones sociales de la Dieta Mediterránea a partir de los discursos de dietistas y mujeres legas de nacionalidad española que residen en la ciudad de Barcelona (Cataluña, España). La comparación de los discursos permite comprender cómo un grupo profesional y un grupo lego manejan e incorporan discursos médicos y sociales en sus prácticas alimentarias. En la modernidad alimentaria, el proceso de medicalización de la alimentación ha contribuido a la emergencia progresiva de una racionalidad científica-nutricional. En este contexto la Dieta Mediterránea emerge en el campo médico encarnando de forma utópica una norma nutricional y moral. Al mismo tiempo, la modernidad alimentaria caracterizada por transformaciones sociales, como la industrialización y la instauración de espacios comunes transnacionales, ha provocado una especie de nostalgia respecto a ciertas prácticas alimentarias consideradas “tradicionales”. En este contexto, la Dieta Mediterránea gane importancia como una herencia cultural a proteger. En este caso, percibimos que los discursos médicos y sociales se fusionan, se complementan y se confunden, generando nuevas normas, representaciones y prácticas alimentarias.

> Palabras clave: Dieta Mediterránea; alimentación saludable; normas nutricionales; patrimonio alimentario; representaciones sociales.

\author{
1 Université Toulouse Jean Jaures. \\ Toulouse, France. Observatório \\ de la Alimentación, Universidat \\ de Barcelona. Barcelona, España \\ (ma.prata@gmail.com). \\ ORCID: 0000-0002-1517-4981 \\ 2 Observatório de la \\ Alimentación, Universidat de \\ Barcelona. Barcelona, España \\ (ursulaverthein@gmail.com). \\ ORCID: 0000-0002-1304-9642.
}

Recebido em: 20/10/2018
Revisado em: 08/02/2019
Aprovado em: 06/05/2019 


\section{Introducción}

En la modernidad alimentaria, ${ }^{1}$ el proceso de medicalización de la alimentación ha contribuido a la emergencia de una racionalidad científica-nutricional (POULAIN, 2002; GRACIA-ARNAIZ, 2010). Este proceso se inscribe dentro de la tradición de reformas alimentarias de origen principalmente anglo-sajón, de carácter puritano, que no sólo apuntaban a establecer prácticas alimentarias “saludables" en la población, sino que también perseguían un orden social y una pureza moral (LEVENSTEIN, 1998). En este contexto, la dieta mediterránea (DM) ha emergido en la medicina, representando de forma utópica una norma nutricional y moral (FISCHLER, 1996), y como un instrumento de gobernabilidad (SILVA, 2016). Al mismo tiempo, esta modernidad alimentaria caracterizada por transformaciones sociales como la industrialización y la instauración de espacios comunes transnacionales, provocó el surgimiento de una "nostalgia" respecto a determinadas prácticas alimentarias. Además, específicamente en el contexto europeo, la creación de la Unión Europea engendró inquietudes con relación a la perdida de la identidad social. La nostalgia asociada a la "crisis identitaria" suscitó el interés por los patrimonios culturales (POULAIN, 1997; ASCHER, 2005; CONTRERAS, 2005a). En este contexto, la DM se tornó una herencia cultural a proteger. Estos movimientos alrededor del concepto de la DM revelan que discursos médicos y sociales se entremezclan, creando nuevas normas, representaciones y prácticas alimentarias.

La alimentación participa en la construcción de la identidad social (FISCHLER, 1990; POULAIN, 2002). De acuerdo con el "principio de incorporación", en el acto alimentario el individuo no sólo incorpora propiedades nutricionales, sino que también las cualidades simbólicas, morales e intelectuales de los alimentos. Este proceso de incorporación hace parte de la creación de la identidad individual y colectiva, así como también de la alteridad. Dado que la cocina, los alimentos y las maneras en la mesa están determinadas socio-culturalmente, es a través de la incorporación alimentaria que se construye el sentimiento de pertenencia a un grupo determinado (FISCHLER, 1990). Los discursos sobre la DM colaboran en la creación de imaginarios sobre la alimentación, participando así en la construcción de la identidad alimentaria y social de los individuos de las zonas mediterráneas.

En este estudio analizamos la DM a partir de las representaciones sociales. Consideramos que estas constituyen una modalidad de pensamiento práctico 
que se presenta como imágenes y sistemas de referencia que permiten pensar, interpretar, significar y clasificar los fenómenos de la vida, la realidad cotidiana, los individuos, el medio ambiente y las informaciones que en él circulan. Se trata de un conocimiento socialmente elaborado y compartido que incide sobre el comportamiento y la organización social, participando de la construcción social de la realidad de cada grupo. Este conocimiento se construye a partir de nuestras experiencias, de las informaciones, conocimientos y modelos de pensamiento que recibimos y transmitimos por medio de la tradición, la educación y la comunicación social (JODELET, 1984). Nuestro interés por las representaciones sociales reside justamente en su dimensión subjetiva, así también como por el hecho de tratarse de un conjunto de referencias socialmente elaboradas y compartidas.

El estudio compara las representaciones sociales de dietistas y de mujeres legas de nacionalidad española que viven en Barcelona (Cataluña, España). Esta comparación permite comprender como una colectividad profesional y una de legas en la materia incorporan los discursos médicos y sociales sobre la DM, observando cómo influyen las normas nutricionales y los sistemas normativos socioculturales. Los resultados presentados derivan de una investigación doctoral realizada en la Universidad de Barcelona, que a través de los discursos sobre el "comer saludable", buscaba identificar las relaciones entre normas dietéticas y normas socioculturales. Durante la realización del estudio se ha observado que cuando las informantes hablaban del "comer saludable" mencionaban con gran frecuencia la DM, lo que nos ha llevado a profundizar en el análisis de este último concepto. En primer lugar, se presenta la metodología de estudio, para enseguida abordar la construcción del concepto de la DM y finalmente analizar los discursos de las informantes.

\section{Metodología}

Esta investigación utiliza una metodología cualitativa basada en entrevistas individuales semi-estructuradas, método que permite acceder a las representaciones de los actores sociales y a las significaciones que los mismos dan a sus comportamientos. Igualmente, este estudio usa un abordaje comparativo sincrónico entre un grupo que tiene una formación técnica-científica en alimentación, las dietistas, y un grupo considerado como lego por no tener formación en dietética. Las comparaciones permiten el entendimiento de las normas, representaciones y prácticas alimentarias y 
de salud, facilitando la interpretación de la influencia de diferentes sistemas normativos sobre cada grupo sociocultural (MENNELL; MURCOTT; OTTERLOO, 1993).

Focalizamos el estudio en participantes del género femenino, dado que las problemáticas de alimentación son experimentadas distintamente según el género con un mayor impacto sobre las mujeres (CAIRNS; JOHNSTON, 2015). Asimismo, la profesión de dietista es esencialmente femenina. El grupo de estudio se limitó a las dietistas que trabajan en consultorios, sin que se determinaran restricciones de edad o años de experiencia. La búsqueda de las dietistas se realizó a partir de: (a) internet, por medio del uso de palabras claves en Google y a través de páginas web de asociaciones profesionales; (b) personas conocidas que nos facilitaron contactos de dietistas; (c) el método "bola de nieve", por el que dietistas entrevistadas pasaron los datos de otras de sus colegas. Todas las profesionales fueron contactadas por correo electrónico o teléfono, momento en el que se les hizo una breve presentación del estudio y se les preguntó por su disponibilidad para participar.

En el caso del grupo de mujeres sin formación en dietética, se delimitó una franja de edad entre los 18 y 30 años, ya que se trata de una etapa de construcción de la autonomía en general y en particular, de la alimentaria. Además, con el fin de obtener una muestra de jóvenes con un nivel educativo equivalente, sólo se consideró entrevistar estudiantes universitarias. Consideramos relevante que las jóvenes cursasen estudios en variadas disciplinas, siendo el único criterio de exclusión la formación en Nutrición Humana y Dietética. La búsqueda de las mujeres no dietistas se realizó: (a) de manera aleatoria en los distintos campus universitarios de Barcelona; (b) a través de personas conocidas; (c) por medio del método "bola de nieve". En todos los casos las mujeres fueron informadas acerca de los objetivos del estudio y, al confirmar la disponibilidad para colaborar, se programaron encuentros para la realización de entrevistas. Cabe notar que, aunque las dietistas y las mujeres legas residían en Barcelona, no todas ellas tenían como origen esta ciudad o Cataluña.

Entre 2010 y 2014, 47 entrevistas semi-estructuradas fueron realizadas, 32 de ellas con mujeres no dietistas y 15 con dietistas, definiéndose la muestra final a partir de la saturación de los discursos obtenidos. Las entrevistas estuvieron orientadas por un guión en castellano que abordaba temáticas relacionadas con la alimentación, la salud y el cuerpo, alcanzando una duración de entre 45 minutos y 2 horas. Las entrevistas fueron grabadas, transcritas y finalmente analizadas, fase en la cual se 
realizaron lecturas sucesivas para la identificación de las temáticas recurrentes y la construcción de tablas temáticas y por categorías.

Para realizar este estudio los autores solicitaron el consentimiento de las participantes, siguiendo las recomendaciones del Código de buenas prácticas en investigación de la Universidad de Barcelona (2010). Finalmente, cabe señalar que la investigación contó con la aprobación de una comisión de expertos de esta misma institución.

\section{La construcción de un concepto}

El concepto de la DM ha ido en ascenso durante los últimos 60 años, siendo debatido por investigadores de ciencias biológicas, humanas y sociales (GONZÁLEZTURMO, 2012a; MEDINA, 2018), que han propuesto una multitud de definiciones que mezclan connotaciones provenientes tanto del campo médico como del cultural (KEYS, 1995; NESTLE, 1995; WILLETT et al., 1995; BACH-FAIG et al., 2011). El concepto de la DM fue "creado" originalmente por médicos norteamericanos. $\mathrm{Su}$ "descubrimiento" científico se remonta a 1948, cuando Leland Allbaught, epidemiólogo estadounidense asociado a la Fundación Rockefeller, consagró un estudio a los diferentes aspectos de la vida cotidiana de los habitantes de Creta, entre los cuales se encontraba su alimentación (SILVA, 2016). Sin embargo, fue a partir de los ańos 60, con los trabajos realizados por Ancel Keys en el marco del Seven Countries Studies, que la DM fue reconocida como modelo de alimentación "saludable", tal y como la conocemos hoy.

A través de un análisis comparativo entre Estados Unidos, Japón, Italia, Holanda, Finlandia, Grecia y la antigua Yugoslavia, se demostró que la DM presentaba beneficios para la salud cardiaca. De la misma manera que las corrientes higienistas alimentarias de carácter científico-religioso desarrolladas en los Estados Unidos a partir del siglo XX (LEVENSTEIN, 1998), Keys pasó a defender la necesidad de retornar a un estilo de vida activo, a los regímenes alimentarios de las civilizaciones tradicionales y rurales, y a una alimentación pobre en carnes y grasa animal (KEYS; KEYS, 1959; SILVA, 2016). Concretamente, es en 1975, con la publicación del libro How to Eat and Stay Well: The Mediterranean Way, que Keys asocia directamente sus recomendaciones a lo que el mismo llama "dieta mediterránea" (KEYS; KEYS, 1975), otorgando argumentos científicos a discursos que antes se basaban en principios morales o normas sociales. 
Consecuentemente, distintos organismos del campo médico organizaron estudios y encuentros científicos con el fin de profundizar los conocimientos sobre este régimen alimentario y crear un modelo higiénico "perfecto". De ellos derivaron numerosas publicaciones, como, por ejemplo, un número especial en el American Journal of Clinical Nutrition editado por Marion Nestlé. Todo esto contribuyó a institucionalizar el concepto de DM dentro del campo médico, y en su conjunto, estos trabajos colaboraron en difundirlo como un modelo alimentario sano "ideal", que debería ser integrado por los individuos independientemente de su ubicación geográfica y cultural. La pirámide de la DM presentada en 1993 por el epidemiólogo de Harvard, Walter Willett, y actualizada posteriormente en diversas ocasiones, también colaboró para que el concepto se popularizara a nivel mundial (BACHFAIG et al., 2011; DERNINI et al., 2012).

Si bien durante décadas se concentró la atención en la dimensión sanitaria de la DM, a lo largo del tiempo el énfasis en sus aspectos históricos y culturales fue en aumento, siendo "reconstruido" gradualmente como patrimonio cultural. Ocurrió así una transición gradual desde una concepción de la DM puramente material, centrada en los efectos biológicos sobre el cuerpo, a una visión cultural (STANO, 2015).

Durante el primer Foro sobre las Culturas Alimentarias del Mediterráneo celebrado en Italia el 2002, expertos en nutrición y antropólogos se pusieron de acuerdo en considerar la DM y las culturas del Mediterráneo como una zona común que debería ser preservada. Tres años más tarde, en 2005, con motivo del $3^{\circ}$ Foro Euro-mediterráneo "Diálogo entre las civilizaciones y pueblos del Mediterráneo: Las culturas alimentarias”, llevado a cabo en Roma, se inició el proceso de candidatura para el reconocimiento por la UNESCO de la DM. En esta ocasión se redactó el documento The 2005 Rome Call for a Common Action on Food in the Mediterranean, en el que se recoge que la DM, más que un simple régimen alimentario, es un estilo de vida (DERNINI et al., 2012). Según Medina (2018, p. 11), los debates organizados en este Fórum buscaban una posición de consenso con respecto a ese concepto, entendido "más allá de una dieta médica, como un sistema cultural". Este debate interdisciplinario estuvo marcado por múltiples negociaciones, incluyendo discusiones relativas a la denominación del bien. Como indica Medina (2018), los sectores de la salud pública y de la agricultura defendían el uso del término "dieta", mientras que los antropólogos abogaban por el término "alimentación”, considerado como más amplio, integrador y menos ligado al 
universo normativo médico. Si bien hubo un esfuerzo por acercar concepciones médicas y antropológicas (GONZÁLEZ-TURMO; MEDINA, 2012), estos datos revelan que tanto la construcción del concepto de DM - transversal a diferentes disciplinas científicas - como la candidatura a patrimonio de la humanidad en sí misma, presentaron una gran complejidad.

En 2007, los gobiernos español, griego, italiano y marroquí sometieron a la UNESCO una candidatura transnacional para el reconocimiento de la DM como patrimonio inmaterial de la humanidad (DERNINI et al., 2012). Esta candidatura fue rechazada y, en 2010, después de una reformulación, la UNESCO finalmente terminó por aprobarla. En su definición de la DM, la UNESCO destaca exclusivamente las dimensiones socioculturales de producción, distribución y consumo alimentario en el Mediterráneo.

La difusión de la DM tanto como un modelo médico como un patrimonio cultural, fue acompañada de un proceso de mediatización, principalmente en los países relacionados con la candidatura (STANO, 2015). MacBeth y Collinson (2004) remarcan que este concepto penetró considerablemente en las creencias populares de números países del sur de Europa, revelando la fuerza de las comunicaciones realizadas en el marco de su promoción. Además, la DM ha conocido una existencia social desconectada del contexto cultural de su "descubrimiento" (SILVA, 2016). Finalmente, cabe notar que estudios recientes (DONINI et al., 2016; DERNINI; BERRY, 2015) asocian la DM a la sostenibilidad, atribuyéndole valores éticos, ecológicos y económicos. En resumen, podemos ver que el concepto va ganando otras acepciones y valores, asumiendo nuevas perspectivas y discursos.

A pesar de todo esto, el concepto de DM también ha recibido críticas. Contreras (2008) afirma que las cocinas de la zona mediterránea son sinónimo de diversidad cultural, reflejan la evolución de sus culturas y comparten una complejidad proveniente de diferentes registros: de las prácticas, conocimientos, habilidades, identidades, preferencias gustativas, etc., por lo que estas herencias difícilmente podrían ser reagrupadas en un conjunto único. Para Montanari (2005, citado por CONTRERAS, 2008), las cocinas son dinámicas y transformar una de ellas en una prescripción sanitaria conllevaría a inmovilizarlas y considerarlas como algo sencillo, asociado a la "naturaleza" de los lugares. Macbeth y Collinson (2004), por su parte, denuncian los intereses económicos y comerciales implicados en la promoción de la DM. 
Fischler (1996) analizó la dimensión "mágica” y "utópica” de la DM. El autor remarca la forma en que los discursos del campo médico relativos a este régimen alimentario, mencionan periódicamente aspectos que van más allá de la nutrición, considerando argumentos extra-científicos. De acuerdo con Fischler, los discursos proponen la idea de un modelo alimentario milenario preconizando de manera utópica un retorno a los orígenes, y sostiene que una reflexión basada en datos históricos revela que la continuidad y la unidad de un régimen mediterráneo son problemáticas. Además, para Fischler:

La historia de la idea del régimen mediterráneo se inscribe en la propensión utopisante de querer reformar la sociedad reformulando las costumbres alimentarias. [...] El "Mediterráneo" es una construcción que encarna progresivamente en forma utópica los ideales político-morales subyacentes (FISCHLER, 1996, p. 11, traducción nuestra).

Según el autor, se trata de una norma moral ideal, lo que le lleva a criticar la intención de considerar tal régimen como un modelo normativo que debe tener alcance "universal". Así, la DM es un concepto que se posiciona en la intersección del mundo médico y de la cultura. Si bien algunos elementos de su definición se refieren a la composición nutricional o a los beneficios que tiene en términos de salud, sobre todo en referencia a las enfermedades crónicas, otros elementos enfatizan su dimensión cultural.

Pese a su auge a lo largo de las últimas décadas, la mayoría de los trabajos sobre ese concepto abordan aspectos epistemológicos, sin considerar las representaciones de los profesionales de la salud y los individuos legos. Este artículo busca así llenar este vacío a partir del análisis de los discursos de estos dos grupos.

\section{Las representaciones sociales de la dieta mediterránea: "salud" y "tradición"}

Según Díaz-Méndez y Gómez-Benito (2010), en España el proceso de patrimonialización de la DM se unió y alimentó de la tendencia a valorizar las cocinas y los productos locales, que surgió en la década de 1980 como parte de la "crisis identitaria" y del deseo de defender la industria local, principalmente, en respuesta a la entrada del Estado español en la Unión Europea. En forma paralela se consolidó la delgadez como norma corporal y algunos productos asociados a la DM fueron percibidos como "buenos" para el control del peso. En este contexto, los discursos sobre la DM fueron incorporados a los mensajes de las políticas de salud 
pública en materia de nutrición (DÍAZ-MÉNDEZ; GÓMEZ-BENITO, 2010). Por ejemplo, la Estrategia NAOS desarrollada a partir de 2005, resalta que la DM posee elementos saludables y que su promoción debe ser una prioridad (AESAN, 2005). Este discurso fue interiorizado por los españoles, influenciando la construcción de las representaciones relativas al acto alimentario y a la "alimentación saludable" (ODELA, 2004). De acuerdo con el Odela, la rapidez con la cual la población hizo propios estos discursos se explica por los mensajes difundidos a través de diversos medios de comunicación y por el cuerpo médico:

Cabe decir que los mensajes de carácter generalista relativos a la bondad de la "DM" y a
su identificación con algunos productos "propios" del paisaje mediterráneo y "propios",
también, de los habitantes de esa región han arraigado profundamente en las mentes de
los espańoles y espańolas que han llevado a cabo una regla de tres directa: "Nosotros
somos mediterráneos, pues comemos DM"; "la DM es sana, nosotros comemos sano"
(ODELA, 2004, p. 106).

Ese concepto ha sido interiorizado por las dietistas y mujeres legas entrevistadas en nuestro estudio. El análisis de sus discursos sobre "alimentación saludable" revela que esta categoría se asocia a un universo normativo y se basa en las nociones de "equilibrio", "moderación" y sobre todo "variedad". Además, las informantes resaltan la importancia de una determinada distribución temporal regular de las comidas. Se ha interiorizado una concepción funcional-nutricional influenciada por la medicalización de la alimentación, concepción que se basa en una perspectiva biológica, técnica y racional, según los principios científicos nutricionales.

Sin embargo, al mismo tiempo las informantes comparten un abordaje psicosocio-cultural que responde a "racionalidades en valor" (POULAIN, 2002), valorando la dimensión hedonista, social y principalmente culinaria del acto alimentario. Además, dan importancia a la calidad de los alimentos en relación a los métodos de producción, cultura y distribución. En sus representaciones, estas características no solo afectan el cuerpo y la salud, sino también al medio ambiente y a los actores sociales. El "alimento sano" es percibido a partir de factores éticos, socioeconómicos y políticos, valorándose distintos aspectos que se confunden en sus representaciones, por ejemplo, los productos percibidos como locales, ecológicos, y, sobre todo, aquellos percibidos como naturales, frescos y de temporada.

La DM es asociada a la "alimentación saludable" y estaría relacionada con factores nutricionales, sociales, culturales, emocionales y simbólicos percibidos como 
inherentes a ella. La DM representa una "alimentación ligera”, lo que en las sociedades lipofóbicas que valoran la delgadez y donde la grasa tiene un status negativo, como en España (GRACIA-ARNAIZ, 2010), acaba siendo un atributo apreciado, asociado a la salud y a la alimentación saludable. La DM también es percibida como más sana porque estaría asociada a ciertos modos de preparación "adecuados", principalmente "a la plancha". Las informantes consideraron esta técnica culinaria junto con "al vapor" y "al horno", como más saludables, sobre todo en oposición a la fritura, ya que son métodos que limitarían el aporte de grasa. Además, aunque la DM no debería ser concebida como un simple modelo nutricional relacionado a una serie de alimentos (GONZÁLEZ-TURMO; MEDINA, 2012), en las representaciones sociales de las informantes, esta dieta aparece asociada a productos alimentarios específicos, sobre todo cereales, verduras, frutas, pescados y aceite de oliva.

Entre las entrevistadas, estos alimentos tienen el status de ser sanos en detrimento de los productos ricos en grasa, azúcar y los industrializados. Estos últimos son percibidos como no frescos, no originarios de su territorio local e inadecuados nutricionalmente, $y$ por ende, menos sanos:

Comer bien es comer variado. La DM creo que es bastante saludable, exactamente las proporciones no sé, pero comer bastante frutas al día, carne dos veces a la semana, pescado, sobretodo también cereales, algo de pasta, arroz, lácteos en menos cantidad (Eli, lega, 19 ańos). ${ }^{2}$

Comer bien es comer verduras, frutas, comer un poco de todo, pero sin excesos. Y la DM que es muy buena, el aceite de oliva, verdura, fruta (Bárbara, lega, 22 años).

Cabe notar la posición del aceite de oliva. Si bien las materias grasas con frecuencia son percibidas como inadecuadas desde un punto de vista médico, el aceite de oliva escapa a estos prejuicios negativos:

En España son buenos los hábitos alimentarios, sobretodo en el Mediterráneo. Comemos muy bien. Para empezar el aceite que es una cosa muy sana y en los otros países no se utiliza tanto y tenemos mucha fruta y verdura, esto hace una alimentación muy buena (Elsa, lega, 21 años).

Las grasas de origen vegetal son mejor valoradas que aquellas de origen animal, principalmente por dos razones que mezclan aspectos simbólicos y nutricionales: la característica "vegetal" asociada a lo "natural", que es percibida positivamente y/o la característica físico-química de las grasas vegetales, normalmente constituidas de ácidos grasos insaturados. Poulain (2002) comprobó, entre individuos franceses, 
que los aceites eran percibidos como las mejores grasas para la salud. Además, respecto a la percepción del efecto de diferentes grasas sobre el peso, los aceites ocupaban la cuarta posición y algunas personas pensaban que el aceite de oliva tenía la virtud de "no engordar".

Según Poulain (2002), estas percepciones no estaban conectadas al contenido real de los lípidos en los productos y la imagen positiva de los aceites habría sido reforzada por el estatus del aceite de oliva. De hecho, en el presente estudio la grasa vegetal mejor valorada es el aceite de oliva, e incluso, en las representaciones de las informantes "pierde" la categoría de producto graso gracias a los discursos positivos que tiene asociado.

Serra-Majem et al. (2007) han estudiado las actitudes alimentarias de individuos catalanes en dos décadas distintas. Mientras en 1992-1993 los alimentos juzgados como muy sanos eran la zanahoria (52\%), la lechuga (48\%), los pescado azules (37\%) y la miel (32\%), en 2002-2003 eran la lechuga (47\%), la zanahoria (46\%), el aceite de oliva (45\%) y los pescado azules (40\%). Según los autores, los resultados obtenidos en la década de 1990 sirvieron para el desarrollo de actividades de educación nutricional que promocionaban el consumo de productos del Mediterráneo, lo que podría haber influenciado las representaciones de la población, principalmente en el caso del aceite de oliva y del pescado azul.

Cabe notar que aunque el aceite de oliva sea uno de los productos más asociados a la DM, tanto históricamente como en la actualidad, no es la única materia grasa consumida en la zona. Como indica González-Turmo (2012b), la reciente popularidad de la DM como modelo nutricional ha colaborado en que se asocie al Mediterráneo con esta única grasa, ocultando el uso de otras como la del cerdo o la mantequilla.

Dado que las dietistas entrevistadas consideran la DM como el "modelo saludable" por excelencia, la integran en sus discursos y prácticas profesionales con el fin de transmitir recomendaciones. Nora (dietista, 27 años) habla de la DM con sus pacientes, asociándola al aceite de oliva:

Hablo del régimen mediterráneo, la gente tiene miedo del aceite de oliva "porque aumenta de peso". No engorda el aceite de oliva, claro, si te pones cantidades enormes si. Les digo "el aceite de oliva es súper importante". El aceite de oliva está comprobado, es súper buen alimento, es súper imprescindible, que lo debería tomar todo el mundo y hay gente que todavía no consume. 
Asimismo, las entrevistadas idealizan el pasado, principalmente rural, como un tiempo en el cual la alimentación era más saludable y lo asocian a la DM. La modernidad alimentaria está caracterizada por numerosas transformaciones, como por ejemplo el proceso de industrialización, los avances tecnológicos, los cambios en las estructuras familiares, las transformaciones del papel de los hombres y mujeres, el aumento del individualismo, etc. (FISCHLER, 1979, 1990; POULAIN, 2002; ASCHER, 2005). Estas transformaciones implican nuevas normas y demandas temporales y espaciales entre los comensales modernos y colaboran con la construcción de un imaginario común relativo a estas dimensiones del comer. Las informantes realizaron una comparación sistemática entre la alimentación "moderna” y la alimentación del pasado, lo que también fue constatado por estudios realizados en otros países (FISCHLER; MASSON, 2008).

De acuerdo con Ascher (2005), en las sociedades basadas en el cambio, el pasado y sus trazos mudan de sentido: el pasado no es solamente lo que continua gracias a la tradición, sino también lo que se pierde. Bessière (2012) habla de la emergencia de un "culto al pasado" en el que las representaciones del pasado estructuran la identidad individual y colectiva, donde el deseo de volver a lo pasado significa (re) identificarse, reencontrar su seguridad, su identidad y su pertenencia a un grupo amenazado por las transformaciones.

Ese tiempo pasado, idealizado, fue evocado por lasinformantes a partir de diferentes ideas e imágenes, usando términos como "originalmente" o "tradicionalmente". Así mismo, hicieron referencia a otras generaciones, a personas mayores, principalmente abuelas, que se ocupaban del trabajo culinario doméstico, que se encargaban de velar por las "buenas" prácticas alimentarias en la familia y que serían testimonios vivos de la "tradición" alimentaria mediterránea. En las representaciones de las entrevistadas, en el pasado los individuos tenían un "estilo de vida más saludable", eran "más activos", estaban "en contacto con la naturaleza” y "comían más sano", su alimentación "era rica en verduras y frutas", y estaba compuesta de alimentos "frescos, naturales y sabrosos". Consideran que la alimentación también era "mejor" porque existía una estructura sociocultural más definida que favorecía las comidas preparadas en casa y consumidas en familia.

Este régimen alimentario "ideal", asociado directamente a la DM, es percibido como un conjunto de costumbres, como su propia "tradición", actualmente en proceso de desaparición: 
Son costumbres que se han perdido... que es muy bueno el tema de la DM que se dice, que es muy rico que tenga las verduras, la fruta (Helena, lega, 25 años).

Cada vez se come peor, creo que antes teníamos la DM típica en España. Era más el producto primario que estaba bueno, se elaboraba poco y se desfrutaba del producto primario. Creo que antes era muy buena, pero creo que ahora con la globalización o no sé si porque la gente tiene menos tiempo o menos ganas de cocinar, cada vez más gente come mal, come muchas cosas precocinadas, además se come en exceso (Ingrid, lega, 23 años).

Según las informantes, esta “degradación” estaría asociada, sobre todo, a un proceso de industrialización y urbanización vinculado con frecuencia a una “invasión” norteamericana, principalmente en la zona urbana:

La comida se está americanizando y la gente come mucho comida rápida, más de lo que debería y la comida mediterránea se está dejando. La gente ahora, los jóvenes, la gente mayor maneja todavía la comida en casa, pero la gente joven normalmente come todo manufacturado y preparado. Tanto Espańa como los países del Mediterráneo tienen una comida más sana. En determinados lugares así de aldea se puede mantener todavía, pero a nivel sociedad, ciudad, está cambiando mucho, se está americanizando mucho (Graciela, dietista, 56 años).

A partir de este prisma, podemos observar que existe una oposición directa entre la DM, percibida como saludable, y un modelo norteamericano considerado como inadecuado. Según Fischler (1996), la DM es un "invento" americano cuyo éxito, principalmente en el "viejo mundo", ha sido paradójicamente alimentado por un espirito anti-americano y por las consecuencias asociadas a los cambios sociales y a la crisis, en un movimiento de reivindicación de una identidad tradicional o nacional.

La DM corresponde así, no solamente a un modelo nutricionalmente perfecto, sino también a uno culturalmente ideal. De hecho, las informantes que asocian este régimen con beneficios a nivel de salud, antes de todo, consideran que es una cuestión de "cultura", de "tradición", un patrimonio a preservar. Tanto las mujeres legas como las dietistas reconocen la DM más como su propia "cultura" que como un régimen de salud "inventado" por médicos. Esta asociación entre su propia cultura alimentaria y la DM es tan importante, que llega al punto de que algunas informantes caracterizan su alimentación a partir de ese concepto, resumiendo toda una serie de productos percibidos como "típicos", prácticas, usos, normas y representaciones que ellas parecen haber integrado en su socialización:

Mi alimentación en 3 palabras sería sana, mediterránea y buena [...] Mediterránea quiero decir productos como el aceite de oliva, las verduras cocinadas a la plancha o las ensaladas, la carne a la brasa o el pescado (Bárbara, lega, 22 ańos). 
Variada, rica en dulces e indirectamente, recorto arroz, pasta, para no engordar... y bastante mediterránea, porque es de productos frescos, aceite de oliva, carnes magras (Alba, dietista, 33 años).

La DM vendría de una transmisión familiar dada de generación a generación, como un patrimonio estructurado a lo largo del tiempo. Graciela (dietista, 56 años) pasó su infancia y adolescencia en Argentina, pero cuando le preguntamos sobre la alimentación que tenían sus padres y si esta había cambiado después de su formación en dietética, explica que sus padres "han conservado el modelo mediterráneo":

No hubo muchos cambios, porque en casa se comía muy bien porque mis padres son espańoles y la comida mediterránea era todos los días entonces era una comida muy sana (Graciela, dietista, 56 años).

En las representaciones de las informantes se considera que la DM es compartida por los habitantes de su zona, siendo revindicada con orgullo, principalmente cuando la comparan a regímenes alimentarios de otros países europeos o de los Estados Unidos, que tendrían una cultura alimentaria más "pesada":

La DM me parece muy buena, porque es comida bastante equilibrada y sana. Cuando estaba en Holanda se ve que se come mucho mejor aquí. Porque en Holanda comen mucho y no tiene algo típico holandés, es algo de todos los sitios, mucho Burger King, McDonald's, salen a comer fuera de esta comida un poco basura. La comida aquí me parece mucho mejor, la DM, es así con las verduras, el aceite de oliva (Vera, lega, 23 ańos).

Al comparar la alimentación entre Holanda y España, Vera expresa que en su país se come "mejor", con lo cual reclama para su identidad nacional una adecuación al modelo mediterráneo atribuyéndole características positivas en comparación con la dieta holandesa. En una comparación que no se basa en elementos objetivos, Vera afirma que en Espańa no se consume fast-food en "gran cantidad" o que en Holanda no hay una comida "típica", lo que no corresponde a la realidad de las prácticas alimentarias de ambos países. En este sentido, la DM no sólo es utilizada para fundamentar la identidad, sino que al mismo tiempo consolida aspectos "positivos" relacionados como la salud y la adecuación a la norma o tradición.

Sin embargo, según algunas informantes, la alimentación mediterránea no sería seguida de manera uniforme en todo territorio español. Aunque haya sido reconocida por la UNESCO como patrimonio de la totalidad del Estado español, para ellas el modelo estaría asociado principalmente a las zonas costeras. Las entrevistadas diferencian la comida de las zonas del litoral, considerada como "sana", de la alimentación de otras partes de España, a las que atribuyen un carácter "pesado”. 
Cabe notar que las informantes originarias de Catalunya son aquellas que presentan este discurso de manera más marcada, mientras que las informantes oriundas de otras regiones, como del País Vasco, consideran que se trata de un modelo compartido por todas las comunidades. Así, la forma en que el concepto es significado varía según la región de origen de cada informante:

En Catalunya menos mal [la alimentación] porque hay la DM y es más sana, pero creo que en el resto de Espańa no, no hay la costumbre de comer una ensalada, un lomo a la plancha (Adaia, lega, 20 años).

Por lo tanto, debemos señalar que la DM se considera un modelo que trae seguridad por su aspecto histórico y anclaje cultural, pero también debido a sus beneficios a nivel de salud, creándose una relación recíproca entre estas dos dimensiones. La imagen de "bueno para la salud" ha alimentado la dimensión "cultural" y, dado que existe una nostalgia y una creencia de que la alimentación "original” es mejor, lo "cultural" alimenta a su vez el aspecto de la "salud", confundiéndose así las virtudes de ambas dimensiones.

De acuerdo con Fischler (1979, 1990), en la modernidad alimentaria, caracterizada por una crisis de los sistemas normativos de regulación social, surgen y se consolidan nuevas formas de gestión del acto alimentario. Tanto la emergencia de discursos sobre alimentación saludable como el deseo de retorno al pasado que se cristaliza entorno al concepto de la DM, se inscriben en la búsqueda de un orden alimentario. Sin embargo, entre estos discursos y la realidad surgen contradicciones.

\section{Paradojas y contradicciones: entre un modelo normativo utópico y la realidad}

Hemos podido observar la existencia de contradicciones y paradojas en los discursos sobre la DM. La primera de ellas surge cuando las informantes valoran la DM como un modelo nutricionalmente y culturalmente "ideal", pero al mismo tiempo critican las normas, prácticas, productos y preparaciones que ellas mismas consideran como parte de la identidad alimentaria de su país y/o región. Las entrevistadas revelan que cuando quieren controlar su alimentación para estar sanas o adelgazar, adaptan o llegan a rechazar ciertos aspectos de su cultura alimentaria. Por ejemplo, Helena (lega, 23 años) pide a sus padres que no hagan paellas, plato que ella designa como "típico" de Barcelona. Julia (lega, 21 años) modificó su alimentación cuando dejó la 
casa de su abuela porque consideraba que comía "demasiado tradicional”, "demasiado pesado". En estos casos, la manera medicalizada de pensar el acto alimentario va en contra de los aspectos de su cultura alimentaria. Existe así una contradicción, ya que las informantes al mismo tiempo critican y valoran los aspectos positivos de su "tradición” alimentaria.

Además, si bien la DM “debería” constituir a priori el modelo alimentario en España, las entrevistadas, principalmente las dietistas, declaran que algunos aspectos de este modelo no están verdaderamente integrados en la alimentación de los españoles. Es por ello, que al momento de dar recomendaciones a sus pacientes y hacerles comprender como la DM se constituye de prácticas "correctas" desde el punto de vista de la nutrición, se basan en los resultados de estudios sobre sus beneficios. Al mencionar los conocimientos científicos que transmite a sus pacientes, Marina (dietista, 35 años) cita la DM y el consumo de frutos secos. Si bien cree que el consumo de frutos secos es una de las características de este régimen, señala que los españoles no tienen la costumbre de comerlos. Es interesante destacar como al referirse sobre este aspecto, Marina se incluye en el discurso utilizando el pronombre "nosotros":

Utilizo un estudio sobre la DM para dar ejemplos, sobre todo cuando hablo de los frutos secos, porque este estudio ha demostrado que un consumo controlado de frutos secos al día es correcto para una alimentación equilibrada. Lo utilizo mucho, porque como no tenemos el hábito de comer frutos secos, cuando comemos frutos secos abusamos, y normalmente son frutos secos fritos o salados, no son crudos.

Así, observamos cómo se presenta una segunda paradoja: si se trata de un modelo compartido en el ámbito cultural "español”, ¿por qué se reconoce que se hace necesario el esfuerzo de difundirlo y consolidarlo entre la población? Parece contradictorio ya que, en teoría, debería ser un patrimonio cultural alimentario consolidado como "allant de soi". Estas constataciones revelan la existencia de una idealización de un modelo alimentario construido, o en las palabras de Fischler, un ejemplo de "utopía" alimentaria (FISCHLER, 1996), percibido como una "tradición intemporal" (SILVA, 2016).

La DM es por tanto un ejemplo de cómo la identidad alimentaria, así como los discursos médicos, son construcciones sociales. De hecho, tanto el patrimonio como la "tradición" son construcciones sociales (CONTRERAS, 2005a; BESSIÈRE, 2012). Valorar el patrimonio significa actualizar, adaptar y reinterpretar aspectos 
desmantelados en la historia de una comunidad, realizando una combinación entre herencia e innovación, seleccionando, clasificando y resaltando valores que dejan (o dejarían) de existir (BESSIÈRE, 2012).

\section{Conclusión}

Observamos como un mismo concepto, el de la DM, ha ido ganando diferentes significados a lo largo del tiempo, según los grupos sociales involucrados y las demandas e intereses impuestos por las transformaciones sociales. En las últimas décadas, la DM ha sido ampliamente difundida, principalmente como resultado de su reconocimiento como patrimonio inmaterial de la humanidad por la UNESCO y por las recomendaciones nutricionales que valoran las características y prácticas asociadas a este modelo. Constatamos que tanto la difusión hecha por el campo médico y las políticas de salud pública, como los discursos derivados del proceso de patrimonialización de la DM, en su conjunto legitiman su existencia.

En este estudio ${ }^{3}$ observamos que tanto las dietistas como las mujeres legas en nutrición, son influenciadas por estos mensajes acerca de la DM. Aunque las profesionales revelen mayor interiorización de las normas científicas nutricionales, sus discursos presentan diversas similitudes con las declaraciones de las mujeres legas, principalmente en relación a los significados que dan a la DM (asociación de sus productos y técnicas con lo "sano", reconocimiento de su repertorio culinario como parte de lo que constituye su identidad, relación de la DM con la "tradición", entre otros). El conjunto de las informantes coincide en representar la DM a través de un modelo nutricional y culturalmente "ideal", como parte constitutiva de "sus propias tradiciones alimentarias" y de sus identidades individuales y colectivas.

Como "creación" del área de la medicina y de la cultura (o del patrimonio), la DM es un ejemplo que revela como campos científicos (medicina, nutrición o ciencias humanas) proponen modelos normativos "utópicos" e intentan encontrar en ellos la solución para cuestiones e inquietudes experimentadas por diversos actores sociales, como la necesidad de auto identificación y la adecuación a las normas de salud o estéticas. A partir del análisis de los discursos de las entrevistadas, pudimos observar cómo estos modelos médicos-nutricionales-culturales producen prácticas y representaciones sociales que participan en la propia construcción y transformación de sus identidades, tanto general como alimentaria, en particular. 
En el discurso de las entrevistadas, pudimos constatar una necesidad de adecuación al modelo de la DM en dimensiones significativas como, por ejemplo, comer "bien", "sano", "justo", "lo propio", y "sostenible". Sin embargo, se ha evidenciado también una falta de correspondencia entre aquello que dicen que se debe comer y aquello que efectivamente comen, con lo que reconocemos una distancia entre las normas alimentarias y sus prácticas efectivas. Por tanto, es en este sentido que se revela lo "utópico" (FISCHLER, 1996) de la DM en su construcción como un modelo alimentario "universal" relacionado directamente con la salud, la cultura y la sostenibilidad, según proponen en la actualidad diferentes campos científicos y profesionales. ${ }^{4}$

\section{Referencias}

AESAN. Agencia Española de Seguridad Alimentaria y Nutrición. Ministerio de Sanidad, Servicio Social e Igualdad. Estrategia para la nutrición, actividad fisica y prevención de la obesidad (Estrategia NAOS). Madrid, 2005.

ASCHER, F. Le mangeur hypermoderne. Paris: Odile Jacob, 2005.

BACH-FAIG, A. et al. Mediterranean diet pyramid today. Science and cultural updates. Public Health Nutrition, v. 14, n. 12, p. 2274-2284, 2011.

BESSIÈRE, J. Patrimoine alimentaire, patrimonialisation. In: POULAIN, J.-P. (Org.) Dictionnaire des cultures alimentaires. Paris: PUF, 2012, p. 978-985.

CAIRNS, K.; JOHNSTON, J. Food and femininity. New York: Bloomsbury Academic, 2015. CONTRERAS, J. Patrimônio e Globalização: o caso das culturas alimentares. In: CANESQUI, A.M.; GARCIA, R.W.D. (org.) Antropologia e Nutrição: um diálogo possível. Rio de Janeiro: Editora Fiocruz, 2005a, p. 129-146.

. El patrimonio alimentario en el área mediterránea. In: TRESSERRAS, J., MEDINA, X. (Orgs.) Patrimonio gastronómico y turismo cultural en el Mediterráneo. Barcelona: IEMed Ibertur, 2008, p. 17-37.

. La modernidad alimentaria. Entre la sobreabundancia y la inseguridad. Revista Internacional de Sociologia, v. 40, p. 109-132, 2005 b.

DERNINI, S. et al. A dietary model constructed by scientists. In: MediTERRA: The Mediterranean Diet for Sustainable Regional Development. Paris: Presses de Sciences Po, 2012, p. $72-88$.

DERNINI, S.; BERRY, E. Mediterranean diet: from a healthy diet to a sustainable dietary pattern. Frontiers in Nutrition, v. 2, s.p, 2015. 
DÍAZ-MÉNDEZ, C.; GÓMEZ-BENITO, C. Nutrition and the Mediterranean diet. A historical and sociological analysis of the concept of a "health diet" in Spanish society. Food Policy, v. 35, p. 437-447, 2010.

DONINI, L.M. et al. A Consensus Proposal for Nutritional Indicators to Assess the Sustainability of a Healthy Diet: The Mediterranean Diet as a Case Study. Frontiers in Nutrition, v.3, s.p., 2016. FISCHLER, C. Gastro-nomie et gastro-anomie. Communications, v. 31, p. 59-61, 1979. . L'Homnivore. Paris: Odile Jacob, 1990.

. Pensée magique et utopie dans la science : de l'incorporation à la diète méditerranéenne. In: (Org.). Pensée magique et alimentation aujourd'hui. Paris: Les Cahiers de l'OCHA, n. 5, 1996. p. 1-17.

FISCHLER, C.; MASSON, E. Manger : français, européens et américains face à l'alimentation. Paris: Odile Jacob, 2008.

GONZÁLEZ-TURMO, I. Régime méditerranéen. In: POULAIN, J.-P. (org.) Dictionnaire des cultures alimentaires. Paris: PUF, 2012a, p. 1127-1137.

, I. The Mediterranean Diet: comsumption, cuisine and food habits. In: CIHEAM. International Centre for Advanced Mediterranean Agronomic Studies. Mediterra 2012. The Mediterranean Diet for Sustainable Regional Development. Paris: Presses de Sciences Po, 2012b.

GONZÁLEZ-TURMO, I.; MEDINA, X. Défis et responsabilités suite à la declaration de la diète méditerranée comme patrimoine culturel immaterial de l'humanité (UNESCO). Revue d'ethnoécologie, [on line], v. 2, s.p, 2012.

GRACIA-ARNAIZ, M. Fat bodies and thin bodies. Cultural, biomedical and market discourses on obesity. Appetite, v. 55, p. 219-225, 2010.

JODELET, D. Représentation social: phénomène, concept et théorie. In: MOSCOVICI, S. (Org.) Psychologie sociale. Paris: PUF, 1984, p. 357-378.

KEYS, A.; KEYS, M. Eat well and stay well. New York: Doubleday, 1959.

. How to Eat Well and Stay Well. The Mediterranean Way. New York: Doubleday, 1975.

KEYS, A. Mediterranean Diet and Public Health: Personal Reflections. American Journal of Clinical Nutrition, v.61, supl., p. 1321S-1323S, 1995.

LEVENSTEIN, H. Dietética contra gastronomia: tradiçóes culinárias, santidade e saúde nos modelos de vida americanos. In: FLANDRIN, J.-L.; MONTANARI, M. (Orgs.) História da Alimentação. São Paulo: Estação Liberdade, 1998, p. 825-840.

MACBETH, H.; COLLISON, P. ¿¿Son nuestras creencias sobre la ciencia racionales? Conceptos sobre una alimentación sana. In: MILLÁN, A. (Org.) Arbitrario cultural: racionalidad e irracionalidad del comportamiento comensal. Homenage a Igor de Garine. Huesca: La Val de Onsera, 2004, p. 405-418. 
MEDINA, X. La construcción del patrimonio cultural inmaterial de carácter alimentario y sus retos en el área mediterránea: el caso de la Dieta Mediterránea. RIVAR, v. 5, n. 14, p. 6-23, 2018. MENNELL, S.; MURCOTT, A.; OTTERLOO, A.H. The sociology of food: eating, diet and culture. London: Sage Publications, 1993.

NESTLE, M. Mediterranean Diets. American Journal of Clinical Nutrition, v. 61, supl., p. 1313S-1320S, 1995.

ODELA. Observatorio de la Alimentación. La alimentación y sus circunstancias: Placer, conveniencia y salud- Estudio. Sobre la realidad de los comportamientos de la población española. V Foro Internacional de la Alimentación. Barcelona: Alimentaria, 2004.

POULAIN, J.-P. Goût du terroir et tourisme vert à l'heure de l'Europe. Ethnologie Française, v. XXVII, n. 1, p. 18-26, 1997.

. Manger aujourd'hui : attitudes, normes et pratiques. Toulouse: Privat, 2002.

SERRA-MAJEM, L. et al. Knowledge, opinions and behaviours related to food and nutrition in Catalonia, Spain (1992-2003). Public Health Nutrition, v. 10, n. 11a, p. 1396-1405, 2007.

SILVA, A. Uma tradição intemporal convertida em ideal alimentar ou o inverso? Acerca da "descoberta científica" da Dieta Mediterrânea. In: PINHEIRO, J.; SOARES, C. (Orgs.) Patrimónios alimentares de aquém e além-mar. Coimbra: Universidade de Coimbra, 2016, p. 23-45.

STANO, S. From nutrients to foods: The alimentary imaginary of the Mediterranean diet. Journal for Communication Studies, v. 8, n. 2, p. 115-132, 2015.

UNIVERSIDAD DE BARCELONA. Código de buenas prácticas en investigación. Barcelona: Agència de Polítiques i de Qualitat UB, 2010.

WILLETT, W. C. et al. Mediterranean Diet Pyramid: a Cultural Model for Healthy Eating. American Journal of Clinical Nutrition, v. 61, supl., p. 1402S-1406S, 1995.

\section{Notas}

${ }^{1}$ Como propone Poulain (2002), usamos el término de modernidad alimentaria para designar lo que pertenece al tiempo alimentario presente. Si consideramos que ese contexto está marcado por profundas transformaciones sociales que corresponden a mutaciones en el campo alimentario, asumimos el uso del término para la descripción de una realidad social compleja y frecuentemente paradójica (FISCHLER, 1990; POULAIN, 2002; ASCHER, 2005; CONTRERAS, 2005b).

${ }^{2}$ Los nombres de las entrevistadas son ficticios.

${ }^{3}$ Agradecimientos: las autoras agradecen el apoyo financiero de la Euroregión Pirineos-Mediterránea y la Fondation Nestlé France.

${ }^{4}$ M. C. M. P. Gaspar definiu o protocolo de pesquisa e realizou a coleta de dados, análise dos dados coletados e redaçáo do artigo. U. Verthein realizou a análise dos dados coletados e a redação do artigo. 


\section{Resumo}

Entre a "saúde" e a "tradiçâo": as representaçóes sociais da dieta mediterrânea

O presente estudo analisa, através de uma abordagem qualitativa, as representaçôes sociais da Dieta Mediterrânea, a partir de discursos de um grupo de nutricionistas e outro de mulheres leigas de nacionalidade espanhola residentes na cidade de Barcelona (Catalunha, Espanha). A comparação dos discursos permite compreender como um grupo profissional e um grupo leigo incorporam discursos médicos e sociais em suas práticas alimentares. $\mathrm{Na}$ modernidade alimentar, o processo de medicalização da alimentação contribuiu com a emergência progressiva de uma racionalidade científica-nutricional. Nesse contexto, a Dieta Mediterrânea se enquadra no campo da medicina, encarnando de forma utópica uma norma nutricional e moral. Ao mesmo tempo, a modernidade alimentar, caracterizada por transformaçóes sociais como a industrialização e a instauração de espaços comuns transnacionais, provocou uma espécie de nostalgia em relação à determinadas práticas alimentares consideradas como "tradicionais". A Dieta Mediterrânea ganha assim importância como uma herança cultural a ser protegida. Percebemos, portanto, nesse caso concreto, que os discursos médicos e sociais se fundem, se complementam e se confundem, criando novas normas, representações e práticas alimentares.

> Palavras-chave: dieta mediterrânea; alimentação saudável; normas nutricionais; patrimônio alimentar; representações sociais. 


\section{Abstract}

Between "health" and "tradition": social representations of the Mediterranean diet

This study analyses, through a qualitative approach, the social representations about Mediterranean Diet of Spanish dieticians and laywomen living in Barcelona (Catalonia, Spain). The comparison between their discourses allows understanding how a professional group and a lay group deal with, and incorporate, social and medical discourses in eating practices. In food modernity, the process of medicalization of food contributed to a progressively emergence of a scientific-nutritional rationality. In that context, the Mediterranean Diet seems to embody, in a utopic way, a nutritional and moral norm. At the same time, this food modernity, characterized by social transformations such as industrialization and the establishment of a common transnational space, provoked a nostalgia concerning some eating practices perceived as "traditional". Therefore, the Mediterranean Diet becomes important as a cultural heritage to be protected. Hence, we may realize that social and medical discourses merge, complement and blend, creating new food norms, representations and practices.

> Keywords: Mediterranean diet; healthy eating; nutritional norms; food heritage; social representations. 